\title{
Coordinated Virtual Inertia Control Strategy for D-PMSG Considering Frequency Regulation Ability
}

\author{
Qiaoming Shi ${ }^{\dagger}$, Gang Wang*, Weiming Ma*, Lijun Fu*, You Wu* and Pengxiang Xing**
}

\begin{abstract}
In the process of virtual inertia control (VIC), the frequency regulation capability of the directly-driven wind turbine with permanent-magnet synchronous generator (D-PMSG) on wind farm is related to its rotor kinetic energy and capacity margin. This paper proposes the method for assessing the D-PMSG frequency regulation capability and defining its coefficient according to the operating state of wind power generators. In addition, the calculating method of parameters in VIC is also discussed according to the principles of primary frequency regulation and inertia response of synchronous generators. Then, by introducing the capability coefficient into the proportion-differential virtual inertia control (PD-VIC) for power coordination, a coordinated virtual inertia control (C-VIC) strategy is developed, with the consideration of the difference in frequency regulation capability between wind power generators. The proposed control method can not only give full play to the frequency regulation capability of wind power generators, decrease the movements of the pitch angle control system but also bring some self-coordination capability to different wind power generators thus to avoid a secondary drop in system frequency. The simulations and experiments prove the proposed method to be effective and practicable.
\end{abstract}

Keywords: Wind turbine generator, Virtual inertia control, Frequency regulation capability, Coordinated control, Frequency response

\section{Introduction}

The D-PMSG on wind farm has gradually become a dominant type of generator in wind power industry because of its low failure rate, high reliability and high power generation efficiency [1]. However, the full-power converter (FPC) in the D-PMSG causes the rotating speed of the permanent-magnet synchronous generator (PMSG) to be decoupled from the frequency of the power grid [2]. As far as its output power and rotating speed are concerned, the D-PMSG is only related to wind speed but fails to respond to the fluctuations of the system frequency under the traditional MPPT control. As a result, it cannot provide inertia and frequency support for the system. With a continuous increase in the injection of wind power, this decoupling effect will add to difficulties in the frequency regulation of traditional synchronous generators only to affect the frequency stability of the system [3].

At present, more and more countries have demanded wind power to provide ancillary services at least like conventional power plants or better according to their grid

$\dagger$ Corresponding Author: College of Electrical Engineering, Xi'an Jiaotong University, China. (shqm14210@163.com)

* National Key Laboratory of Vessel Integrated Power System Technology, Naval University of Engineering, China. (wanggang60742@126.com, maweimin@public.wh.hb.cn, lijunfu2006@sina.cn,wuyou1351@126.com)

** School of Electrical Engineering, Wuhan University, China. (pxxing1990@163.com)

Received: July 6, 2015; Accepted: June 22, 2016 codes [4]. To enable D-PMSG to provide frequency support for the system as traditional synchronous generators do, two control modes have been proposed [4]: power reserve control [5-7] and rotor kinetic energy control [3, 8, 9]. The power reserve control mode is used for reserving power through the adjustment of rotating speed or pitch angle of the wind turbine. This method can improve its frequency response capability, but will affect its power generation efficiency for lack of economical character. The rotor kinetic energy control mode means introducing the auxiliary power related to the proportional and derivative terms of the frequency deviation on the basis of MPPT control. When the system frequency fluctuates, the D-PMSG can adjust the kinetic energy stored in the rotor so as to participate in the inertia response and primary frequency regulation of the system. Therefore, this control mode is also called PD-VIC [3]. PD-VIC enables the wind power generators to be involved in the system frequency response and also guarantees the electricity-generating efficiency. Thus, it has become a focal point of research. The values of the control parameters determine the extent to which the wind power generators participate in the system frequency response in PD-VIC. However, there have been no references found about the calculating method of the control parameters. Therefore, it is necessary to discuss the calculating method of parameters in VIC to provide the basis for further research of the VIC.

Because of the limitation of the capacity of a single wind power generator, investigations into the demand for wind 
power generators participating in the system frequency regulation should be carried out according to the level of a wind farm. At actual wind farm, there are hundreds of wind power generators lying at different locations and running at different wind speeds with the result that their operating states and frequency regulation capabilities are quite different. If an indiscriminate frequency regulation control is conducted, some of the generators will not bring their frequency regulation capability into full play, and some others will quit the frequency response earlier due to the excessive frequency regulation triggering the rotating speed protection, which will cause a secondary drop in system frequency [8]. Therefore, it is necessary to carry out a research on the optimal coordinated control of frequency responses of wind power generators so as to provide considerable and effective power support for the power grid.

To perform a coordinated control of frequency responses of wind power generators, references $[10,11]$ and $[12,13]$ dealt with the power coordinated allocation and the coordinated recovery of wind turbine rotating speed respectively. In the aspect of power coordinated allocation, reference [10] gave a definition of the weight coefficient of frequency regulation power that is proportional to the operational wind speed of the unit based on the pitch angle control, thereby representing the frequency regulation capability of wind power generators. Reference [11] proposed a control method of using different droop coefficients under different wind speed conditions based on the rotating speed reserve control. However, the control modes mentioned in [10] and [11] could be easily affected by the fluctuation and uncertainty of the wind speed used as an input control signal. In the aspect of the coordinated recovery of wind turbine rotating speed, references [12] and [13] suggested realizing the coordinated recovery of rotating speed through time delay response. That approach can avoid a secondary drop in system frequency to some extent, but it is complicated for use at a large-scale wind farm. Furthermore, a discussion into the frequency regulation capacity of D-PMSG was made in [13], but no quantitative analysis method has been proposed for its frequency regulation capability.

In order to maximize the frequency regulation capability of wind power generators and avoid a secondary drop in system frequency caused by a premature or collective withdrawal of the wind power generators from frequency response, using D-PMSG as an object, this paper has made a study of both the method of calculating parameters of VIC and the method of assessing the frequency regulation capability of wind power generators. On this basis, it has proposed a C-VIC method for wind farms in view of the frequency regulation capability of D-PMSGs.

This paper is composed of eight sections. Section 1 gives an introduction about the advances in the research into the frequency response control of D-PMSG. Section 2 and Section 3 describe the fundamental principle of PD-
VIC and calculating method of its control parameters respectively. Section 4 proposes the method for assessing frequency regulation capability and defining frequency regulation capability coefficient of D-PMSG. Section 5 presents the C-VIC method for multi-units on wind farm. Section 6 and Section 7 refer to the effectiveness and feasibility of the proposed method verified by means of simulations and experiment system, respectively.

\section{Principle of Conventional VIC}

The D-PMSG mainly consists of wind turbine, PMSG, and FPC. The wind turbine directly drives the PMSG to generate electricity. The FPC implements the conversion and control of the output power [1]. The system structure of the D-PMSG which has been connected to the grid is shown in Fig. 1.

After the introduction of VIC into D-PMSG, its active power control system has a structure as shown in Fig. 2, in which wind power $P_{\text {wind }}$ captured by the wind turbine is expressed as [14]:

$$
P_{\text {wind }}=0.5 \rho \pi r^{2} v^{3} C_{p}(\lambda, \theta)
$$

where $\rho$ is air density, $r$ is blade radius, $v$ is wind speed, $C_{p}$ is power coefficient, $\theta$ is blade pitch angle, $\lambda$ is tip speed ratio, $\lambda=\omega_{w} r / v$, and $\omega_{w}$ is the rotating speed of the wind turbine.

The conventional PD-VIC makes the D-PMSG change its output when the system frequency fluctuates mainly through the introduction of the auxiliary power related to the proportional and derivative terms of the system frequency deviation so as to simulate the properties of primary frequency regulation and inertia response of the traditional synchronous generators. The auxiliary power $P_{V I C}$ can be expressed as follows [3]:

$$
P_{V I C}=-k_{p} \Delta \omega^{*}-k_{d} p \Delta \omega^{*}
$$

where $p$ is the differential operator; $k_{p}$ and $k_{d}$ are the proportional and derivative parameters respectively; $\Delta \omega^{*}$ is the per unit value of system frequency deviation; $\Delta \omega^{*}=\omega^{*}$ $\omega_{n}{ }^{*}, \omega^{*}=\omega / \omega_{n}, \omega_{n}{ }^{*}=1 ; \omega$ is the angular frequency of the system; $\omega_{n}$ is the rated angular frequency of the system.

The reference power $P_{w_{-} \text {ref }}$ of the wind power generators

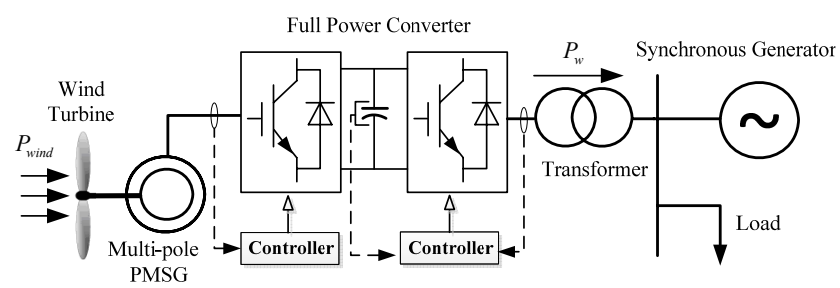

Fig. 1. The structure of wind power integrated system 


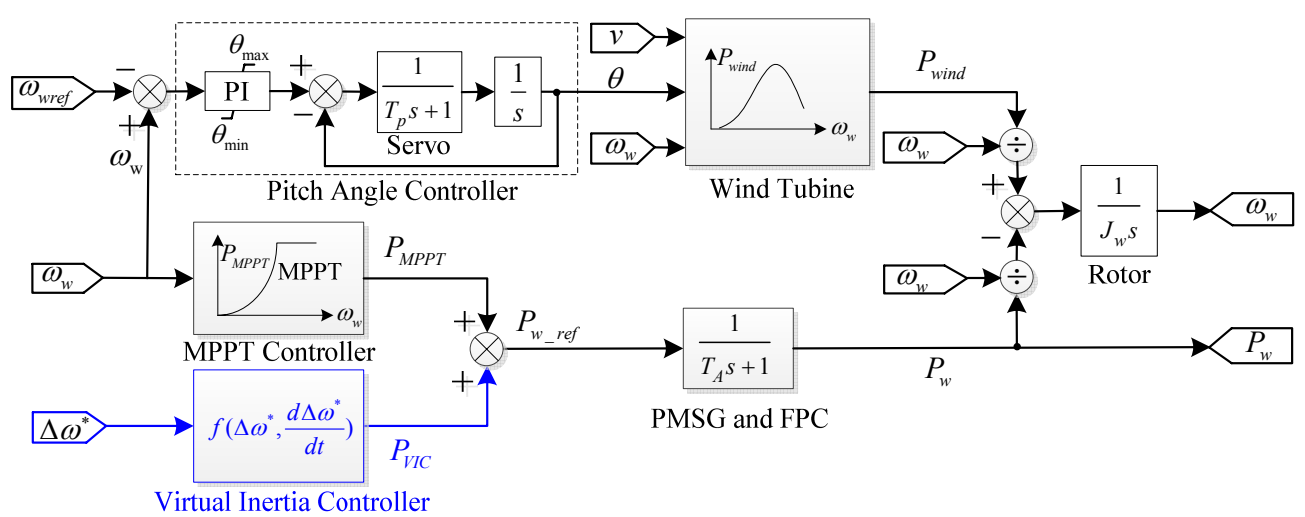

Fig.2. The structure of D-PMSG active power control system

includes MPPT control output power $P_{M P P T}$ and VIC auxiliary power $P_{V I C}$, namely

$$
P_{w_{-} r e f}=P_{M P P T}+P_{V I C}
$$

where $P_{M P P T}$ is expressed as $[1,15]$ :

$$
P_{M P P T}=k_{\max } \omega_{w}^{3}
$$

where $k_{\max }$ is the coefficient used for the wind turbine to obtain the maximum energy, and its numerical value is $0.5 \rho \pi r^{5} C_{\text {pmax }} / \lambda_{\text {opt }}^{3}$, in which $C_{\text {pmax }}$ and $\lambda_{\text {opt }}$ are respectively the power coefficient and tip speed ratio corresponding to the optimal power.

The D-PMSG implements a closed-loop tracking control of reference power through FPC. For the sake of simplified analysis, it is possible to ignore the electromagnetic loss of the unit and simplify the PMSG and FPC as an inertia part $[3,16]$. As a result, the grid-connected power $P_{w}$ of the DPMSG can be expressed as:

$$
P_{w}=\frac{1}{T_{A} S+1} P_{w_{-} r e f}
$$

where $s$ is the Laplace operator; $T_{A}$ is the time constant of the PMSG and FPC, whose value is about 0.2s [3]. The frequency response process is an electromechanical transient process, which lasts a few seconds, so the dynamic properties of FPC can be ignored, then the expression is

$$
P_{w}=P_{w_{-} r e f}
$$

According to (3) and (6), the equation for the dynamic state of the rotor in the D-PMSG is expressed as follows:

$$
J_{w} p \omega_{w}=\frac{P_{w i n d}}{\omega_{w}}-\frac{P_{M P P T}+P_{V I C}}{\omega_{w}}
$$

where $J_{w}$ is the rotational inertia of the D-PMSG.

The principle of the pitch angle control system of D-
PMSG is shown in Fig. 2. The pitch angle controller is a PI controller. $T_{p}$ is the inertia time constant of the servo motor in the pitch angle control system [11]. When the wind speed is low, the pitch angle remains $\theta_{\min }$ to maximize the captured wind energy. When the larger wind speed causes the rotating speed to exceed the rated value, the pitch angle control system will increase the pitch angle and decrease the mechanical power captured by the wind turbine, thereby reducing the rotating speed.

\section{Calculating Method of VIC Parameters}

The frequency regulation auxiliary power $P_{\mathrm{VIC}}$ can be divided into $P_{1}$ and $P_{2}$ in PD-VIC, which are used for simulating the characteristics of primary frequency regulation and inertia response of the synchronous generators respectively. $P_{1}$ and $P_{2}$ are expressed as

$$
\begin{gathered}
P_{1}=-k_{p} \Delta \omega^{*} \\
P_{2}=-k_{d} p \Delta \omega^{*}=-k_{d} p \omega^{*}
\end{gathered}
$$

The D-PMSG can be regarded as a virtual equivalent synchronous generator in calculating the parameters in VIC. The equivalent synchronous generator has the same rated capacity as the D-PMSG, with its mechanical speed $\omega_{\text {vir }}$ completely coupled with system frequency $\omega$, namely $\omega_{v i r}=\omega / p_{0 e}$, where $p_{0 e}$ is the number of pole pairs of the equivalent synchronous generator. The concrete parameters of the D-PMSG and the equivalent synchronous generator are: for the D-PMSG, its rated capacity is $P_{w n}$, its rated rotating speed is $\omega_{w n}$, its rotational inertia is $J_{w}$ and its inertia time constant is $H_{w}$, while for the equivalent synchronous generator, its droop coefficient is $R$, its rotational inertia is $J_{v i r}$, its inertia time constant is $H_{v i r}$, its rated rotating speed is $\omega_{\text {virn }}=\omega_{n} / p_{0 e}$ and its rated power is $P_{\text {virn }}=P_{w n}$.

The primary frequency regulation and inertia response capability of the equivalent synchronous generators is related to its droop coefficient $R$ and inertial time constant 
$H_{v i r}$, respectively. Here are the calculating methods of $k_{p}$ and $k_{d}$.

\subsection{Calculating method of $k_{p}$}

The droop coefficient $R$ of the equivalent synchronous generator is [17]:

$$
R=-\frac{\Delta \omega^{*}}{\Delta P_{G} / P_{v i r n}}
$$

where $\Delta P_{G}$ is the static deviation of output power of the equivalent synchronous generator.

Considering $P_{\text {virn }}=P_{w n},(10)$ is expressed as:

$$
\Delta P_{G}=-\frac{P_{w n}}{R} \Delta \omega^{*}
$$

The following expression can be obtained based on the equivalent relation between the D-PMSG and the equivalent synchronous generator:

$$
P_{1}=\Delta P_{G}
$$

$k_{p}$ is obtained from (8), (11)-(12):

$$
k_{p}=\frac{P_{w n}}{R}
$$

It is shown in (13) that $k_{p}$ is related to the rated capacity and droop coefficient of the units; the smaller $R$ is, the larger $k_{p}$ is.

\subsection{Calculating method of $\boldsymbol{k}_{d}$}

The D-PMSG has the same kinetic energy variation as the equivalent synchronous generator over a period of $\Delta t$ in the process of VIC. The expression is as follows:

$$
\begin{aligned}
& \Delta E_{k}=\frac{1}{2} J_{w}\left[\left(\omega_{w 0}+\Delta \omega_{w}\right)^{2}-\omega_{w 0}{ }^{2}\right] \\
& =\frac{1}{2 p_{0 e}{ }^{2}} J_{v i r}\left[\left(\omega_{0}+\Delta \omega\right)^{2}-\omega_{0}^{2}\right]
\end{aligned}
$$

where $J_{v i r}$ is the rotational inertia of the equivalent synchronous generator, or the rotational inertia shown by the D-PMSG in the power grid under VIC. $\omega_{w 0}$ and $\Delta \omega_{w}$ denote the initial value and variation of the mechanical angular speed respectively, $\Delta \omega_{w}=\omega_{w}-\omega_{w 0}, \omega_{0}$ and $\Delta \omega$ represent the initial value and variation of the system electromagnetic angular frequency respectively, and $\Delta \omega=\omega-\omega_{0} . J_{v i r}$ can be derived from (14) with the second order small quantity $\Delta \omega_{w}{ }^{2}$ and $\Delta \omega^{2}$ ignored.

$$
J_{v i r}=p_{0 e}{ }^{2} J_{w} \frac{\omega_{w 0}}{\omega_{0}} \frac{\Delta \omega_{w}}{\Delta \omega}
$$

The inertia time constant of the equivalent synchronous generator is [17]:

$$
H_{v i r}=\frac{J_{v i r} \omega_{n}{ }^{2}}{2 p_{0 e}{ }^{2} P_{v i r n}}
$$

With $P_{\text {virn }}=P_{w n}$ taken into account and according to (15) and (16), the following expression can be obtained.

$$
H_{v i r}=\frac{J_{w} \omega_{w n}{ }^{2}}{2 P_{w n}} \frac{\omega_{n}{ }^{2}}{\omega_{w n}{ }^{2}} \frac{\omega_{w 0}}{\omega_{0}} \frac{\Delta \omega_{w}}{\Delta \omega}
$$

Also, $H_{w}$ can be expressed as:

$$
H_{w}=\frac{J_{w} \omega_{w n}^{2}}{2 P_{w n}}
$$

Therefore, from (17) and (18) $H_{v i r}$ is obtained:

$$
H_{v i r}=\frac{\omega_{w 0}^{*}}{\omega_{0}^{*}} \frac{\Delta \omega_{w}^{*}}{\Delta \omega^{*}} H_{w}
$$

where $\omega_{0}{ }^{*}=\omega_{0} / \omega_{n}, \omega_{\mathrm{w} 0}{ }^{*}=\omega_{w 0} / \omega_{w n}, \Delta \omega_{\mathrm{w}}{ }^{*}=\Delta \omega_{w} / \omega_{w n}$.

The virtual inertia coefficient $\gamma$ is defined as:

$$
\gamma=\frac{\omega_{w 0}{ }^{*}}{\omega_{0}{ }^{*}} \frac{\Delta \omega_{w}^{*}}{\Delta \omega^{*}}
$$

So, $H_{v i r}$ can be expressed according to (19) and (20):

$$
H_{v i r}=\gamma H_{w}
$$

It can be seen from (21) that the rotational inertia produced by the D-PMSG in the power grid is no longer zero under VIC but related to the inherent inertia $H_{w}$ and virtual inertia coefficient $\gamma$ of the D-PMSG. The value of $\gamma$ reflects the amount of virtual inertia of the D-PMSG and $\gamma$ is adjustable. When $\gamma<1$, the rotational inertia produced by the D-PMSG in the power grid is less than its own inherent inertia; when $\gamma>1$, the D-PMSG provides greater virtual inertia than its own inherent inertia for the power system. The high value of $\gamma$ will make the wind power generators release the kinetic energy too quickly in an actual system, which will lead the units to quit the frequency response too early.

In the practical operation, the fluctuation of frequency is less than $0.02 \mathrm{pu}(1 \mathrm{~Hz})$ when the most serious fault occurs in the system, and meanwhile the minimum rotating speed of wind power generators under VIC is higher than $0.6 \mathrm{pu}$. As a result, the value ranges of four variables in (20) are generally as following: 


$$
\left\{\begin{array}{l}
\omega_{w 0}{ }^{*} \in(0.60,1.00) \\
\omega_{0}{ }^{*} \in(0.98,1.02) \\
\Delta \omega_{w}{ }^{*} \in(-0.40,0.40) \\
\Delta \omega^{*} \in(-0.04,0.04)
\end{array}\right.
$$

Therefore, the value range of $\gamma$ is approximately $[0,10]$ based on (22).

Without the proportional control taken into consideration, the characteristics of the D-PMSG in VIC can be expressed as follows:

$$
2 H_{w} p \omega_{w}{ }^{*}=\frac{P_{w i n d}{ }^{*}}{\omega_{w}{ }^{*}}-\frac{P_{M P P T}{ }^{*}}{\omega_{w}{ }^{*}}-\frac{P_{2}^{*}}{\omega_{w}{ }^{*}}
$$

where $\mathrm{P}_{\text {wind }}=\mathrm{P}_{\text {wind }} / \mathrm{P}_{\mathrm{wn}}, \mathrm{P}_{\mathrm{MPPT}}{ }^{*}=\mathrm{P}_{\mathrm{MPPT}} / \mathrm{P}_{\mathrm{wn}}, \mathrm{P}_{2}{ }^{*}=\mathrm{P}_{2} / \mathrm{P}_{\mathrm{wn}}$.

When the system is under steady-state operation, $P_{2}^{*}=0$ and $P_{\text {wind }}{ }^{*}=P_{M P P T}{ }^{*}$ for the D-PMSG; when the system is subjected to large load disturbances, the virtual inertial controller will be activated. At the beginning of its action, (23) can be expressed as:

$$
2 H_{w} p \omega_{w}^{*}=\frac{-P_{2}^{*}}{\omega_{w}{ }^{*}}
$$

Furthermore, (19) can be expressed as:

$$
H_{v i r}=\frac{\Delta \omega_{w}^{*} / \Delta t}{\Delta \omega^{*} / \Delta t} \frac{\omega_{w 0}^{*}}{\omega_{0}^{*}} H_{w} \approx \frac{p \omega_{w}^{*}}{p \omega^{*}} \frac{\omega_{w}^{*}}{\omega^{*}} H_{w}
$$

Combining (24) and (25) and considering $\omega \stackrel{*}{\approx} 1, P_{2}=P_{2}{ }^{*}$ $P_{w n}$, we can get:

$$
P_{2}=-2 H_{v i r} P_{w n} p \omega^{*}
$$

$k_{d}$ can be obtained according to (9), (21) and (26):

$$
k_{d}=\gamma k_{d 1}
$$

where $k_{d 1}$ is the constant and its value is $k_{d 1}=2 H_{w} P_{w n}$.

\section{Assessment of Frequency Regulation Capability of Wind Power Generator}

Fig. 3 shows the curve of the D-PMSG operating in a static mode when wind speed changes $[1,18,19]$. In Fig. 3, the operation range is divided into four zones: Zone1 to Zone4. In Zone1, the D-PMSG does not start working. Zone4 represents the pitch angle control region. Zone2 and Zone3 represent MPPT control regions, and meanwhile the Zone 3 is also a VIC region.

In VIC, the frequency regulation capability of each D-

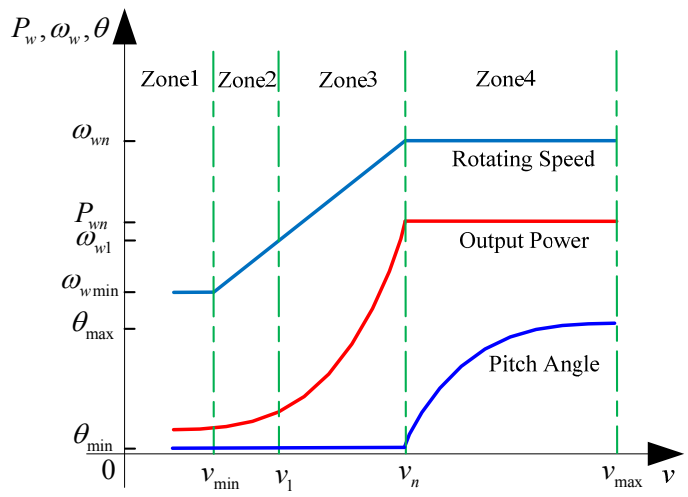

Fig. 3. Characteristics curves of D-PMSG under operation

PMSG on wind farm is related to its rotor kinetic energy and capacity margin. When the system frequency rises and drops, the rotor kinetic energy and capacity margin will have different effects on the frequency response of wind power generators. So it is necessary to evaluate the frequency regulation capability of each one operating when the system frequency rises and drops.

\subsection{During a drop in system frequency}

When there is a drop in system frequency due to a sudden rise in load or a sudden loss of generator power, the wind power generators need to increase their power output and release their rotor kinetic energy to participate in system frequency regulation. At this time, the wind power generators in a high wind speed area have relatively high rotor kinetic energy. But because of the capacity limitation of converters, the auxiliary power they can supply for frequency regulation is low. The wind power generators in a low wind speed area have large capacity margin, but the auxiliary power they can provide for frequency regulation is also low due to slow rotating speed and less rotor kinetic energy. The wind power generators in a medium wind speed area have both considerable rotor kinetic energy and larger capacity margin, so the auxiliary power they can supply for frequency regulation is relatively high.

When the system frequency drops, the rotor kinetic energy which can be released by a wind power generator represents the quantity of frequency regulation resources it possesses. Therefore, the available rotor kinetic energy factor $k_{J 1}$ of wind turbines can be defined as follows:

$$
k_{J 1}=\frac{E_{k}-E_{k 1}}{E_{k n}-E_{k 1}}
$$

where $E_{k n}, E_{k}$, and $E_{k 1}$ are the kinetic energy of a wind power generator running respectively at rated rotating speed $\omega_{w n}$, current rotating speed $\omega_{w}$, and the lower limit of rotating speed protection $\omega_{\mathrm{w} 1}$. And the value of $E_{k}$ is:

$$
E_{k}=0.5 J_{w} \omega_{w}^{2}
$$


As shown in (28), the higher the rotor kinetic energy $E_{k}$ is, the greater the available rotor kinetic energy factor $k_{J 1}$ is.

Wind power generators cannot run at too low a speed in the process of frequency regulation, otherwise they cannot be restored to a normal operation state. In the rotating speed protection module, when a wind power generator runs at a speed lower than the lower limit $\omega_{w 1}$, it will go away from frequency regulation. Usually $\omega_{w 1}$ is set to be 0.6 times the rated value, or $\omega_{w 1}=0.6 \omega_{w n}$.

In the running process of D-PMSG, the closer the maximum extracted power $P_{M P P T}$ is to the capacity limit of the converter $P_{w n}$, the smaller the value space of auxiliary power for frequency regulation $P_{V I C}$ is. To represent the frequency regulation potential of wind power generators, the available capacity factor $k_{C 1}$ is defined as follows:

$$
k_{C 1}=\frac{P_{w n}-P_{w}}{P_{w n}-P_{w 1}}
$$

where $P_{w n}, P_{w}$, and $P_{w 1}$ represent the grid-connected power of a wind power generator running respectively at rated rotating speed $\omega_{w n}$, current rotating speed $\omega_{w}$, and the lower limit of rotating speed protection $\omega_{\mathrm{w} 1}$. And the value of $P_{\mathrm{w} 1}$ is $P_{w 1}=0.6^{3} P_{w n}=0.216 P_{w n}$, which means that the wind power generator will not participate in frequency response when its power output is lower than $0.216 \mathrm{pu}$. As shown in (30), the higher the power output of the wind power generator is, the smaller the available capacity factor $k_{C 1}$ is.

When the system frequency drops, the frequency regulation capability coefficient $k_{a 1}$ of wind power generators can be defined as the per unit value of the product of available rotor kinetic energy factor $k_{J 1}$ and available capacity factor $k_{C 1}$, namely

$$
k_{a 1}=\left\{\begin{array}{l}
\frac{k_{J 1} \times k_{C 1}}{\left[k_{J 1} \times k_{C 1}\right]_{\max }}, \omega_{w} \in\left[\omega_{w 1}, \omega_{n}\right] \\
0, \omega_{w} \in\left(\left[0, \omega_{w 1}\right) \cup\left(\omega_{n},+\infty\right]\right)
\end{array}\right.
$$

where $\left[\bullet_{\max }\right.$ means getting the maximum value. The expression $\left[k_{a 1}\right]_{\max }=1$ is obtained from (31).

For the D-PMSG, from (4) and (28)-(31) the following formula can be derived.

$$
k_{a 1}=7.099\left(a^{2}-0.6^{2}\right)\left(1-a^{3}\right)
$$

where $a=\omega_{w}{ }^{*}, a \in[0.6,1]$, and $\left[k_{J 1} \times k_{C 1}\right]_{\max }=0.281$.

\subsection{During a rise in system frequency}

When the system frequency rises due to a sudden loss of load, the wind power generators participate in system frequency regulation by reducing the active power output and increase the rotor kinetic energy on the basis of MPPT control. At this time, the wind power generators in a high wind speed area have high adjustable converter capacity, but their rotating speed is close to the rated value. Reducing power output is likely to make the rotating speed exceed the rated value thus to cause an action of pitch angle. For the wind power generators in a low wind speed area, there is much space for the rotating speed to rise, but the adjustable capacity of the converter is relatively low. So the participation in frequency regulation by the wind power generators should be avoid, otherwise their low power output will cause the system to be out of stability. The wind power generators in a medium wind speed area have both considerable frequency regulation capacity and relatively large space for the rotating speed to rise. And so their frequency regulation capability is higher.

When the system frequency rises, the active power which can be reduced by a wind power generator represents the quantity of frequency regulation resources it possesses. The available capacity factor $k_{C 2}$ is defined as follows:

$$
k_{C 2}=\frac{P_{w}-P_{w 1}}{P_{w n}-P_{w 1}}
$$

As is shown in (33), the higher power output a wind power generator has, the larger its available capacity factor is.

Frequent action of the pitch angle control system will shorten the service life of wind power generator. However, in a high wind speed area, the participation in frequency regulation by wind power generator tends to cause frequent motion of the pitch angle control system. To avoid this consequence, there is need for considering the amount of kinetic energy which a wind power generator can actually absorb. For the frequency regulation potential of wind power generators, the available rotor kinetic energy factor $k_{J 2}$ is defined as follows:

$$
k_{J 2}=\frac{E_{k n}-E_{k}}{E_{k n}-E_{k 1}}
$$

It shows that the higher the rotor kinetic energy is, the smaller the available rotor kinetic energy factor is.

When the system frequency rises, the frequency regulation capability coefficient $k_{a 2}$ of wind power generators can be defined as the per unit value of the product of available rotor kinetic energy factor and available capacity factor, namely

$$
k_{a 2}=\left\{\begin{array}{l}
\frac{k_{C 2} \times k_{J 2}}{\left[k_{C 2} \times k_{J 2}\right]_{\max }}, \omega_{w} \in\left[\omega_{w 1}, \omega_{n}\right] \\
0, \omega_{w} \in\left(\left[0, \omega_{w 1}\right) \cup\left(\omega_{n},+\infty\right]\right)
\end{array}\right.
$$

For the D-PMSG, from (4) and (33)-(35), the following formula can be obtained.

$$
k_{a 2}=9.018\left(a^{3}-0.6^{3}\right)\left(1-a^{2}\right)
$$




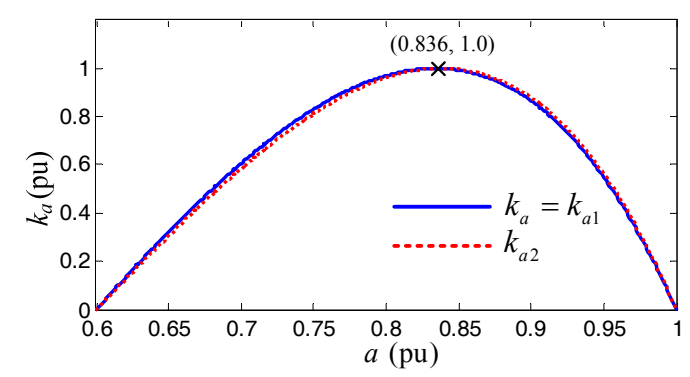

Fig. 4. Curves of frequency regulation capability coefficients of D-PMSG

where $a \in[0.6,1]$ and $\left[k_{J 2} \times k_{C 2}\right]_{\max }=0.221$.

As shown in Fig. 4, the characteristic curves of the DPMSG's frequency regulation capability are derived from (32) and (36). In Fig. 4, the blue full curve and red dashed curve respectively represent a change in the coefficient of frequency regulation capability when the system frequency drops and rises, and the basic coincidence of the two curves indicates that under different load disturbance conditions, the frequency regulation capability coefficients of the D-PMSG are basically the same though the defining methods are different. In actual control, (32) is used to simplify the control method, without the necessity for detecting the type of variation in system frequency. Therefore, the frequency regulation capability coefficient $k_{a}$ of wind power generators can be defined as follows:

$$
k_{a}=k_{a 1}
$$

When $a \in[0.6,1], k_{a}$ merely increases first and then decreases accordingly with the rise of the rotating speed of the wind power generator. When $a=0.836, k_{a}$ acquires the maximum value. It is obvious that the frequency regulation capability comprehensively expresses the frequency regulation capacity and the rotor kinetic energy.

\section{C-VIC Method for Multi-Units on Wind Farm}

\subsection{Principle of C-VIC}

As far as the traditional PD-VIC method is concerned, the control parameters are adjusted mainly according to the rated operation state of the D-PMSG, which cannot represent their real-time status. In order to give full play to the frequency regulation capability of each wind power generator on wind farm and meanwhile avoid its too much participation in frequency regulation, the introduction of the frequency regulation capability coefficient into the traditional PD-VIC and the utilization of (13) and (27) can result in obtaining a C-VIC method for the real-time frequency regulation ability. The expression is as follows:

$$
P_{V I C}=k_{a}\left(-k_{p} \Delta \omega^{*}-\gamma k_{d 1} p \omega^{*}\right)
$$

where $k_{a}$ represents the frequency regulation capability a wind power generator actually has and the extent to which it participates in frequency regulation. The larger the value of $k_{a}$, the greater frequency regulation capability of wind power generators and the more contribution to frequency regulation. $k_{a}$ is related to operating state of wind power generators and their operating states are different from one another. Under the condition of multi-unit integrated grid, the introduction of $k_{a}$ enables the wind power generators to automatically adjust the extent of their participation in frequency regulation according to their own frequency regulation capability, thereby implementing the coordinated VIC.

\subsection{Application of C-VIC}

In Fig. 5 the concrete structure diagram of the C-VIC is shown. In Fig. 5 (a), $U_{d c n}$ is the rated DC bus voltage of the FPC, the system frequency $\omega^{*}$ is obtained through software phase locked loop (SPLL) [20], it should be a low-pass filtered in the differential calculation because of the high frequency harmonics involved in $\omega^{*}$ [4]. In C-VIC, frequency modulation ability coefficient $k_{a}$ is calculated based on (37), $k_{p}$ and $k_{d 1}$ are calculated based on (13) and (27) respectively.

In the system frequency response process, when the rotating speed drops to $0.6 \mathrm{pu}$, the rotating speed protection module makes the wind power generators temporarily withdraw from frequency regulation by setting flag1 to zero. This will help restore the wind power generators to a safe rotating speed and prevent them from halting due to extremely low rotating speed. The amplitude-limited module can ensure that the power output of wind power generators will not exceed the capacity limitation of the converter.

When grid fault happened in AC system, voltage of the wind power grid connected point will be affected and drop at a certain scope, the D-PMSG should not only stay in grid by low voltage ride through but also provide advisable reactive power to the grid to support the voltage recovery [21]. In this interval, grid side converter of the D-PMSG can't output active power to the grid normally because of the current limit, resulting in a high voltage in DC bus of the converter. Low voltage ride through control can consume redundant active power by crowbar circuit when DC bus voltage exceeds its limit [22]. In order to avoid extra burden on crowbar circuit brought by C-VIC when grid faults happen in $\mathrm{AC}$ system, the $\mathrm{AC}$ system fault protection module will set flag2 to 0 to exit C-VIC when DC bus voltage exceed $1.05 U_{d c n}$ before crowbar circuit is motivated. When the fault in AC system is cleared, the voltage of DC bus will return to normal and flag2 will be reset to 1 by the $\mathrm{AC}$ system fault protection module to restart C-VIC. Because grid faults in AC system usually last a very short time (about $100 \mathrm{~ms}$ ), exit of C-VIC has less effect on the system frequency during grid fault. 


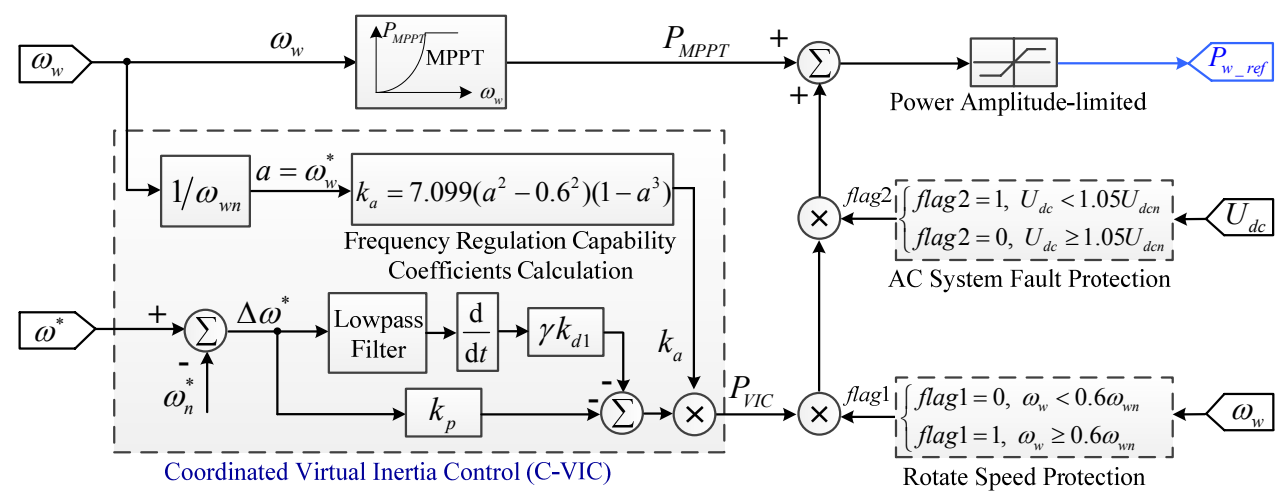

(a) C-VIC structure of D-PMSG

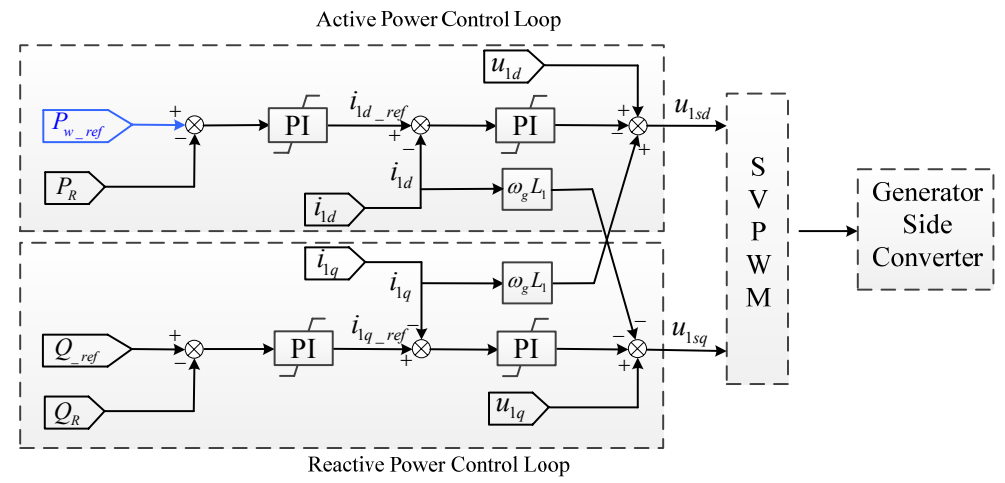

(b) Control scheme of generator side converter

Fig. 5. Full control for D-PMSG generator side converter with C-VIC

The control scheme of the generator side converter is shown in Fig.5 (b). The control scheme is composed of active power control and reactive power control, both of them adopt double loop control and the feed-forward decoupling control is adopted to eliminate the coupling of $d$-axis variable and $q$-axis variable in the inner loop [14]. In Fig. 5 (b), $P_{R}$ and $Q_{R}$ are the input active power and reactive power, $u_{1 d}$ and $u_{1 q}$ are the $d$-axis and $q$-axis voltage measured at the terminal of PMSG, $i_{1 d}$ and $i_{1 q}$ are the $d$-axis and $q$-axis current measured at the same position respectively, $L_{1}$ is the filter inductance, $\omega_{g}$ is the angular frequency of generator side voltage, $u_{1 s d}$ and $u_{1 s q}$ are the $d$ axis and $q$-axis control voltage respectively.

\section{Simulation analysis}

To verify the effectiveness of the C-VIC method for wind farms, a simulation test system is set up in Matlab/ Simulink simulation platform, as shown in Fig. 6. It involves an equivalent wind farm and an equivalent thermal power station. The former contains 30 D-PMSGs, each with the rated capacity of is $2 \mathrm{MW}$. The latter contains a synchronous generator with the installed capacity of 60 MW. For the convenience of comparison, the 30 D-PMSGs at the wind farm are divided into 3 groups, each with 10 generators. The wind speeds for 3 groups are set as $11 \mathrm{~m} / \mathrm{s}$,

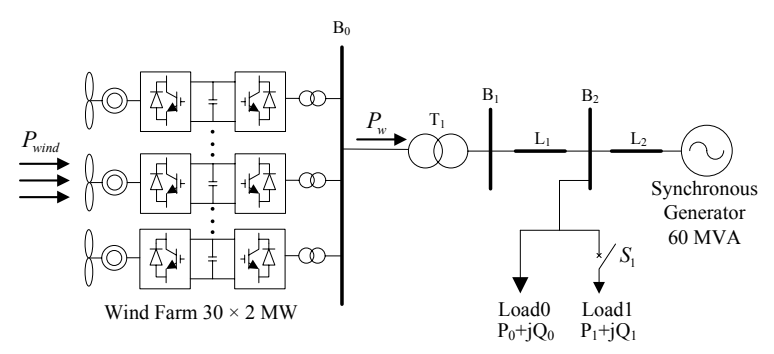

Fig. 6. Single line diagram of simulation test system

$9.5 \mathrm{~m} / \mathrm{s}$ and $7.5 \mathrm{~m} / \mathrm{s}$, which respectively represent high, medium and low wind speed. The system load includes a constant load (load0) and a switched load (load1). $S_{1}$ is used for the switching of load1. As for the modeling of the simulation system, refer to [11]. The parameters of the simulation system are offered in Appendix A.

According to (13) and (27), the VIC parameters $k_{p}$ and $k_{d 1}$ of D-PMSG are $k_{p}=2.0 \times 10^{6} / 0.04=5.0 \times 10^{7}$ and $k_{d 1}=$ $2 \times 2.0 \times 10^{6} \times 6.0=2.4 \times 10^{7}$, where $R=0.04, P_{w n}=2.0 \times 10^{6} \mathrm{~W}$, and $H_{w}=6.0 \mathrm{~s}$. In the traditional PD-VIC, $\gamma$ is set as 0.58 to prevent the wind power generators from participating excessively in frequency regulation under the condition of low wind speed as the value of $\gamma$ is too large. In the $\mathrm{C}$ VIC, the participation of wind power generators in frequency regulation in the case of low wind speed is restricted because of introducing the frequency regulation capability coefficient, while the wind power generators 
display more virtual inertial in the condition of medium and high wind speed. So the value of $\gamma$ can be increased to $\gamma=2.32$. The rotating speed protection value is set as $\omega_{w 1}=0.6 \omega_{w n}$. The expression for the frequency regulation capability coefficient is $k_{a}=7.099\left(a^{2}-0.6^{2}\right)\left(1-a^{3}\right)$.

\subsection{A sudden increase in system load}

The system load is set as $P_{0}=70 \mathrm{MW}$ and $P_{1}=15 \mathrm{MW}$. The initial state of the switch $S_{1}$ is an open state. When $t=$ $10 \mathrm{~s}, S_{1}$ is closed and the system load increases by $15 \mathrm{MW}$. Then the system is simulated respectively in the three modes of MPPT control (without VIC), traditional PD-VIC, and C-VIC. The frequency response curves of the system are compared, as shown in Fig. 7. The numbers (1), (2), and (3) denote the wind power generators in high, medium, and low wind speed areas, respectively.

As shown in Fig. 7, when the system frequency drops due to a sudden increase in load, the rotating speed and power output of D-PMSG do not respond to this change under MPPT control. The lowest point of system frequency reaches $49.30 \mathrm{~Hz}$. The introduction of PD-VIC leads to a significant improvement in the system frequency characteristic, with the lowest point up to $49.71 \mathrm{~Hz}$ and its time delay from $11.60 \mathrm{~s}$ to $13.12 \mathrm{~s}$. However, the auxiliary power which each wind power generator supplies is equal because the actual frequency regulation capability of each wind power generator is not take in account. Due to their low frequency regulation capability, the wind power generators in the low wind speed area are forced to quit the frequency regulation at $12.28 \mathrm{~s}$ when their rotating speed drops to the lower limited value of rotating speed protection. As a result, there is a secondary drop in system frequency. At the same time, the frequency regulation capability of wind power generators in the high wind speed area cannot be brought into full play, so that their rotating speed varies only in a small range.

Compared with PD-VIC, the C-VIC can further increase the lowest point of system frequency to $49.79 \mathrm{~Hz}$, and the time of reaching the lowest point is extended from $11.60 \mathrm{~s}$ to $15.45 \mathrm{~s}$. It is seen from Fig. 7 (d) that in C-VIC, the capability coefficient $k_{a}$ of the wind power generators varies with their operating conditions. By automatically adjusting the frequency regulation ability coefficient, the wind power generators in the medium and high wind speed areas are fully involved in frequency regulation, whereas the wind power generators in the low wind speed area avoid excessive participation. Moreover, the mark " $\times$ " in Fig. 7(c) indicates that under C-VIC the wind power generators no longer enter the rotating speed recovery state simultaneously. The wind power generators in the low, medium and high wind speed areas have an interval up to 5 $\mathrm{s}$ for their rotating speed restoration, which significantly weakens the secondary impact of the rotating speed recovery on the system frequency.

\subsection{A sudden decrease in system load}

The system load is set as $P_{0}=55 \mathrm{MW}$ and $P_{1}=15 \mathrm{MW}$. The initial state of $S_{1}$ is a closed state. At $t=10 \mathrm{~s}$, the switch $S_{1}$ turned on to reduce the system load from $70 \mathrm{MW}$ to 55 MW. The simulation tests for the system are carried out
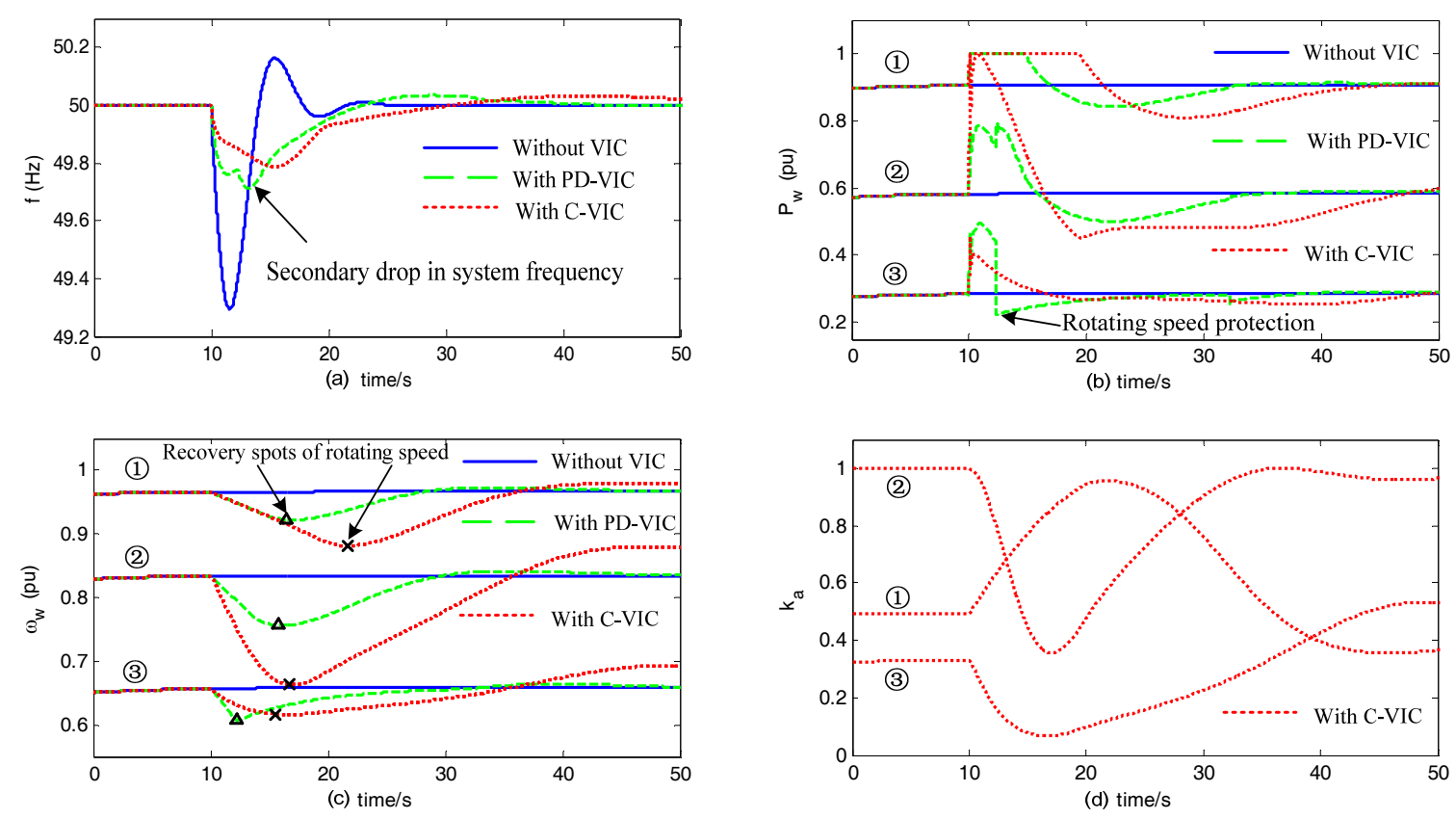

Fig. 7. Comparison of system responses in the increase of system load: (a) Characteristic curves of system frequency response; (b) Power curves of D-PMSGs connected to the grid; (c) Rotating speed curves of D-PMSGs; (d) Curves of frequency regulation capability coefficients in C-VIC 

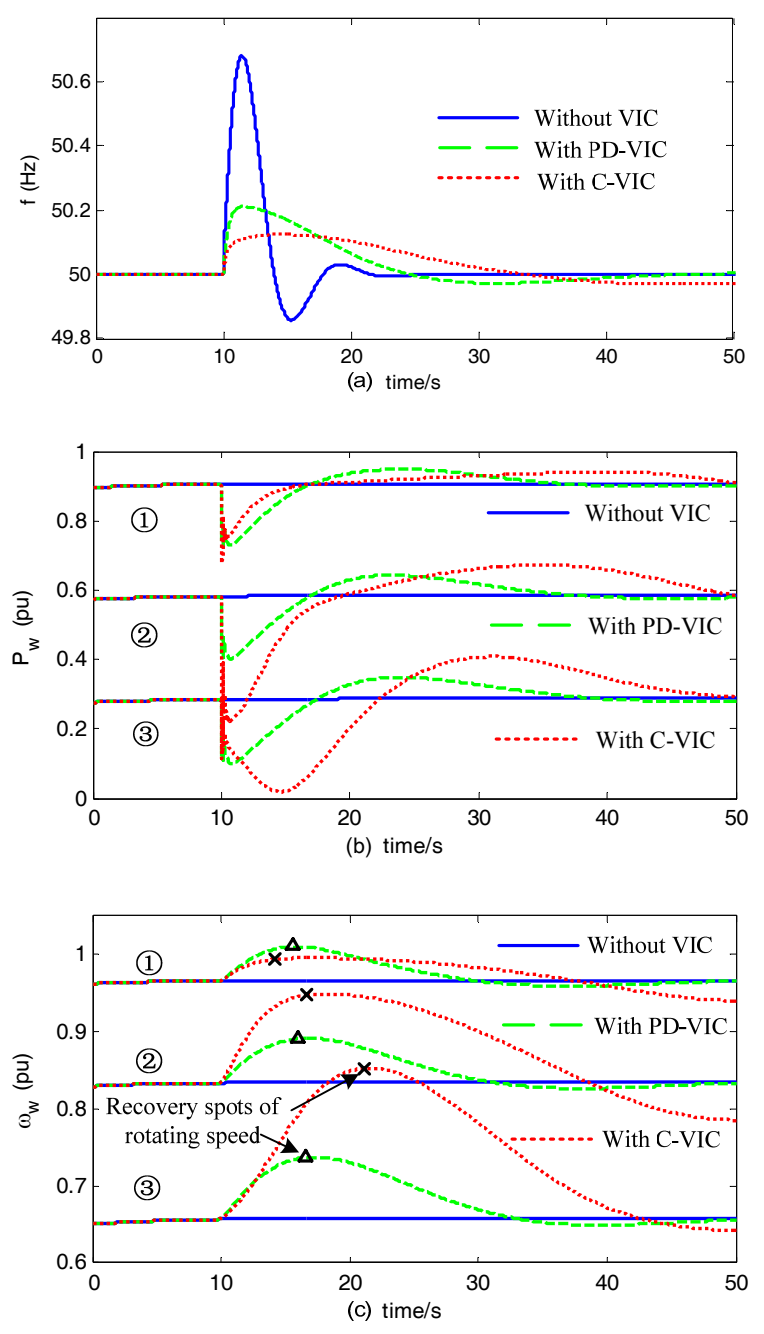

respectively under MPPT control (without VIC), traditional PD-VIC, and C-VIC. In Fig. 8, the frequency response curves of the system in three different control modes are shown. The numbers (1), (2), and (3) denote the wind power generators in high, medium, and low wind speed areas, respectively.

As shown in Fig. 8, when the system frequency rises due to a sudden decrease in load, the traditional PD-VIC will make the maximum frequency of the system decline from $50.68 \mathrm{~Hz}$ to $50.21 \mathrm{~Hz}$ and the time to reach the peak point is extended from $11.47 \mathrm{~s}$ to $11.62 \mathrm{~s}$. However, the auxiliary power supplied by each wind power generator is equal because this control mode does not involve the actual frequency regulation capability of each wind power generator. As shown in Fig. 8(c)-(d), when the wind power generators in the high wind speed area run so fast as to reach the rated speed, the pitch angle control system is triggered to act. What is worthy of note is that the frequent action of the pitch angle control system will influence the service life of wind power generators.

After the C-VIC is used, the maximum value of system frequency further falls to $50.13 \mathrm{~Hz}$, and the time of reaching the maximum value is extended from $11.47 \mathrm{~s}$ to
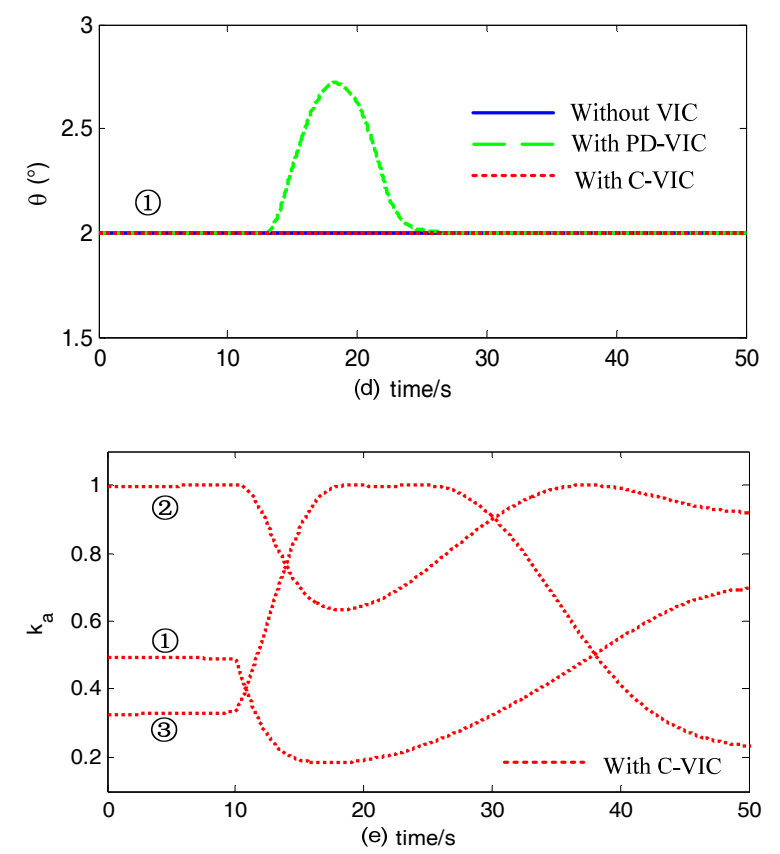

Fig. 8. Comparison of system responses in the decrease of system load: (a) Characteristic curves of system frequency response; (b) Power curves of D-PMSGs connected to the grid; (c) Rotating speed curves of D-PMSGs; (d) Pitch angle curves of D-PMSGs in high wind speed areas; (e) Curves of frequency regulation capability coefficients in C-VIC.

14.50 s. Compared with the traditional PD-VIC, the C-VIC is capable of bringing into full play the frequency regulation capability of the wind power generators in the low wind speed area by adjusting the coefficient $k_{a}$ automatically while the output power does not decline too much. Moreover, it can prevent the wind power generators in the high wind speed area from participating in frequency regulation to such an extent that the pitch angle control system may act. Besides, the mark " $\times$ " in Fig. 8 indicates that under $\mathrm{C}-\mathrm{VIC}$, the wind power generators in different wind speed areas do not simultaneously go into a rotating speed recovery state any more.

To sum up, the C-VIC of wind power generators functions well in frequency regulation in the conditions of a sudden increase or a sudden decrease in the system load. In the C-VIC mode, the wind power generators in different wind speed areas can automatically participate in frequency regulation according to their ability to perform a multi-unit coordinated control. In this case, the wind power generators operating in different states can restore their rotating speed in turn, thereby lessening a secondary effect on system frequency.

\section{Experimental Verification}

In order to further verify the effectiveness and feasibility 


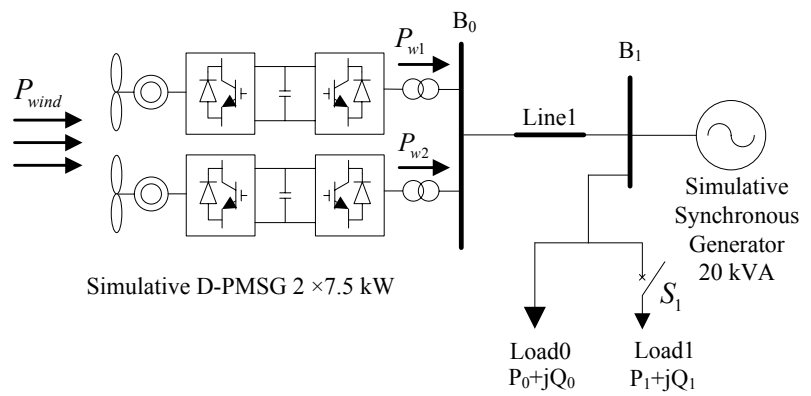

Fig. 9. Principle structure of experiment system

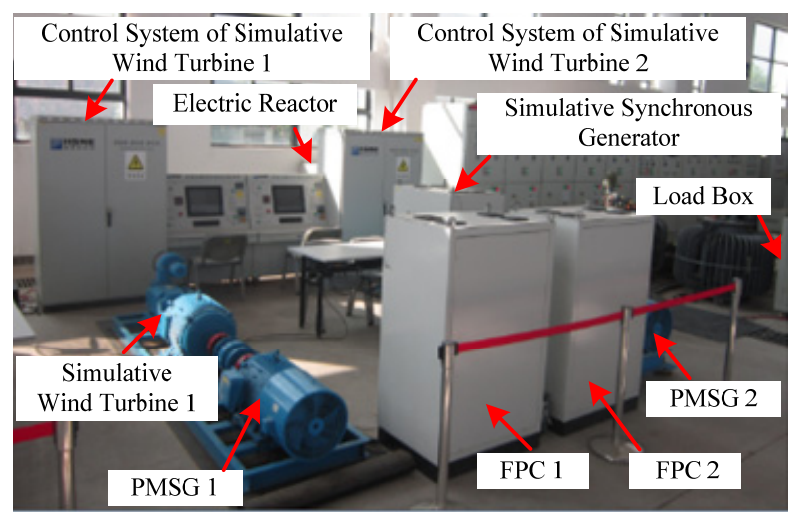

Fig. 10. Distribution of field experiment devices

of the proposed C-VIC strategy for D-PMSGs, a $35 \mathrm{kVA}$ experimental system has been constructed to simulate wind power integration, as shown in Fig. 9. The experimental system is mainly composed of two $7.5 \mathrm{~kW}$ simulative DPMSGs and a $20 \mathrm{kVA}$ simulative synchronous generator. As for the simulative D-PMSGs, the way to simulating the wind turbines is similar to what is mentioned in [18] and [19]; that's a DC motor is driven by the DC speed regulator to simulate the characteristics of wind turbine and the output is controlled by a FPC. In the experimental system, the simulation of the synchronous generator is implemented by the inverter mainly according to the principle of virtual synchronous $[23,24]$. The system load depends on a resistance load box for simulation and the long-distance transmission line 1 depends on an electric reactor for simulation. The distribution of the field experiment devices is shown in Fig. 10, and the detailed parameters of the experiment system are offered in Appendix B.

From (13) and (27) we can get the VIC parameters of the simulative D-PMSG: $k_{p}=7.5 \times 10^{3} / 0.04=1.88 \times 10^{5}$ and $k_{d 1}=$ $2 \times 7.5 \times 10^{3} \times 5.7=8.55 \times 10^{4}$, where $R=0.04, P_{w n}=7.5 \times 10^{3} \mathrm{~W}$, and $H_{w}=5.7 \mathrm{~s}$. In the traditional PD-VIC mode, $\gamma$ is 0.5 . In the $\mathrm{C}$-VIC mode, $\gamma=2.00$. The rotating speed protection value is set as $\omega_{\mathrm{w} 1}=0.6 \omega_{\mathrm{wn}}$. The expression for the frequency regulation capability coefficient is $k_{a}=7.099\left(a^{2}\right.$ $\left.0.6^{2}\right)\left(1-a^{3}\right)$.

The constant wind speeds of $9.5 \mathrm{~m} / \mathrm{s}$ and $7.0 \mathrm{~m} / \mathrm{s}$ are set respectively for simulative D-PMSGs 1 and 2 to represent

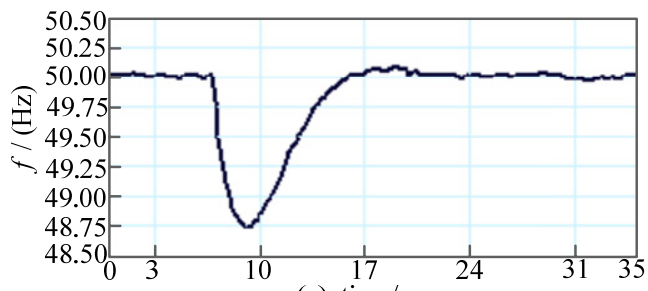

(a) time/s

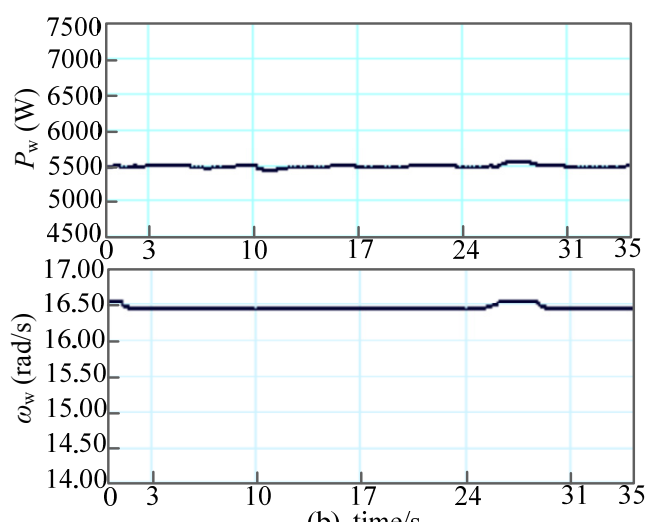

(b) time/s

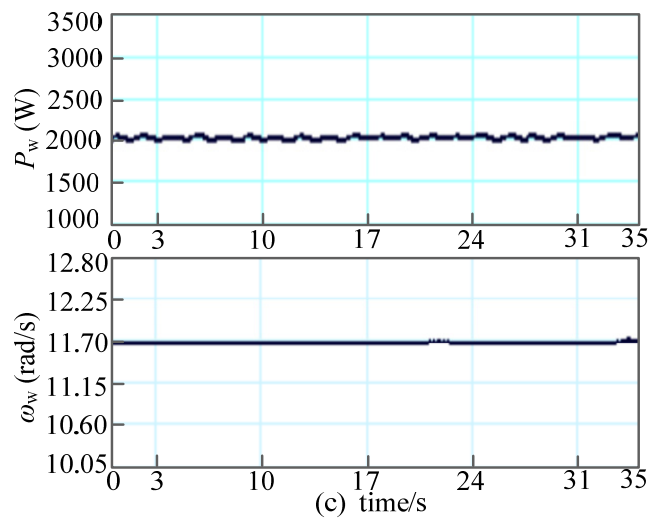

Fig. 11. System response characteristic curves without VIC introduced: (a) Characteristic curve of system frequency response; (b) Curves of rotating speed and output power of Simulative D-PMSG 1; (c) Curves of rotating speed and output power of Simulative D-PMSG 2.

those of wind power generators in two different wind speed areas at an actual wind farm. The initial load of the system is $20 \mathrm{~kW}$ and then a load disturbance of $4.3 \mathrm{~kW}(21.5 \%)$ is added to the system suddenly. At this time experimental tests and comparisons are carried out respectively without VIC, with traditional PD-VIC and with C-VIC.

\subsection{Without VIC introduced}

When VIC is not introduced, the wind power generators work in the MPPT control mode. The system response characteristic curves are shown in Fig. 11.

As shown in Fig. 11, the experimental results are similar to the simulation results in Fig. 7. When a sudden disturbance is added to the load in the system, two 
simulative D-PMSGs into which VIC is not introduced almost keep the rotating speed and output power unvarying under constant wind speed condition and do not respond to variation in the system frequency. At this time, the frequency regulation of the system completely depends on the simulative synchronous generator. The system frequency has a large range of fluctuation and its lowest point reaches $48.75 \mathrm{~Hz}$.

\subsection{With PD-VIC introduced}

With the traditional PD-VIC introduced, the characteristic curves of system frequency response appear, as shown in Fig. 12, similar to the simulation results in Fig. 7. The introduction of traditional PD-VIC into the system leads

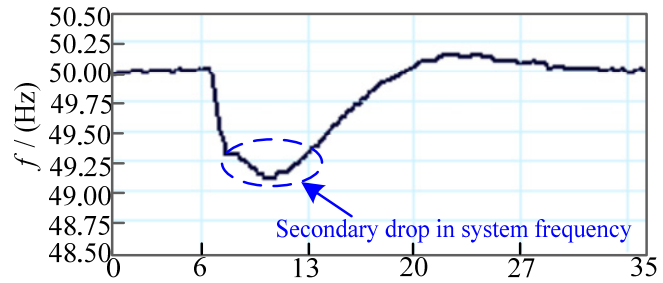

(a) time $/ \mathrm{s}$

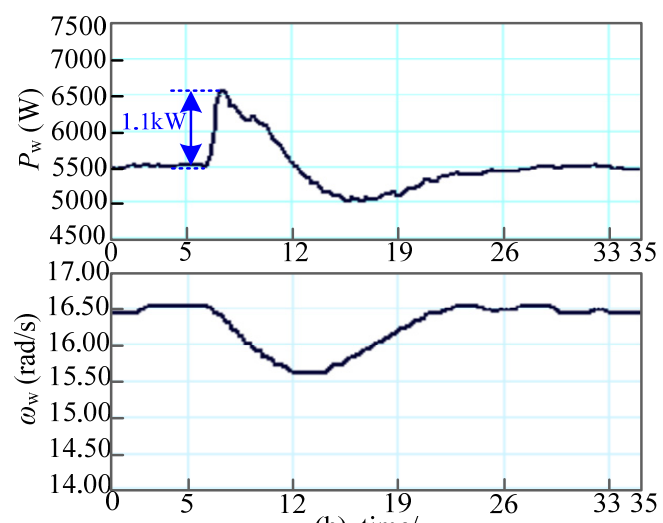

(b) time/s

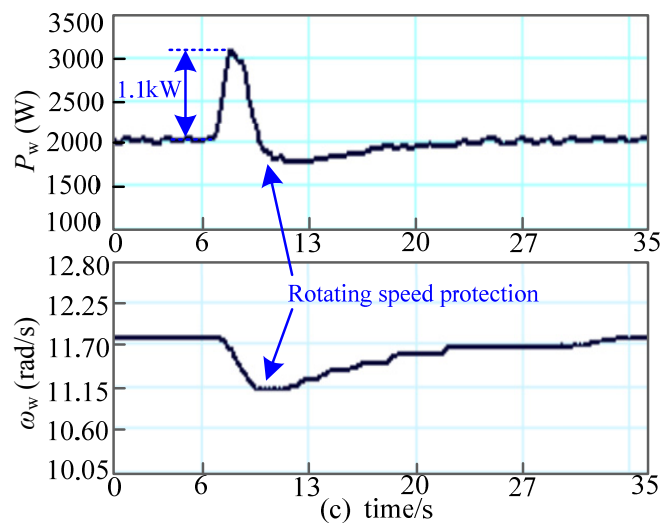

Fig. 12. System response characteristic curves with PDVIC introduced: (a) Characteristic curve of system frequency response; (b) Curves of rotating speed and output power of Simulative D-PMSG 1; (c) Curves of rotating speed and output power of Simulative D-PMSG 2. the lowest frequency to rise from $48.75 \mathrm{~Hz}$ to $49.12 \mathrm{~Hz}$, but the auxiliary frequency regulation powers of the two simulative D-PMSGs are the same, with the maximum

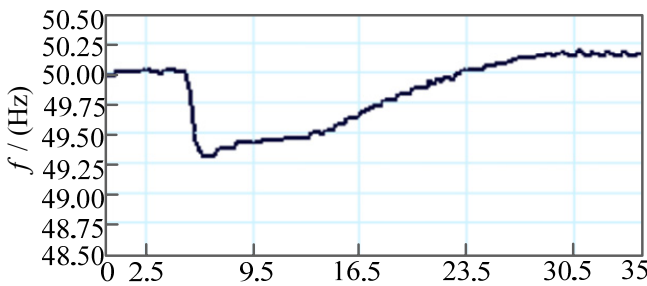

(a) time/s
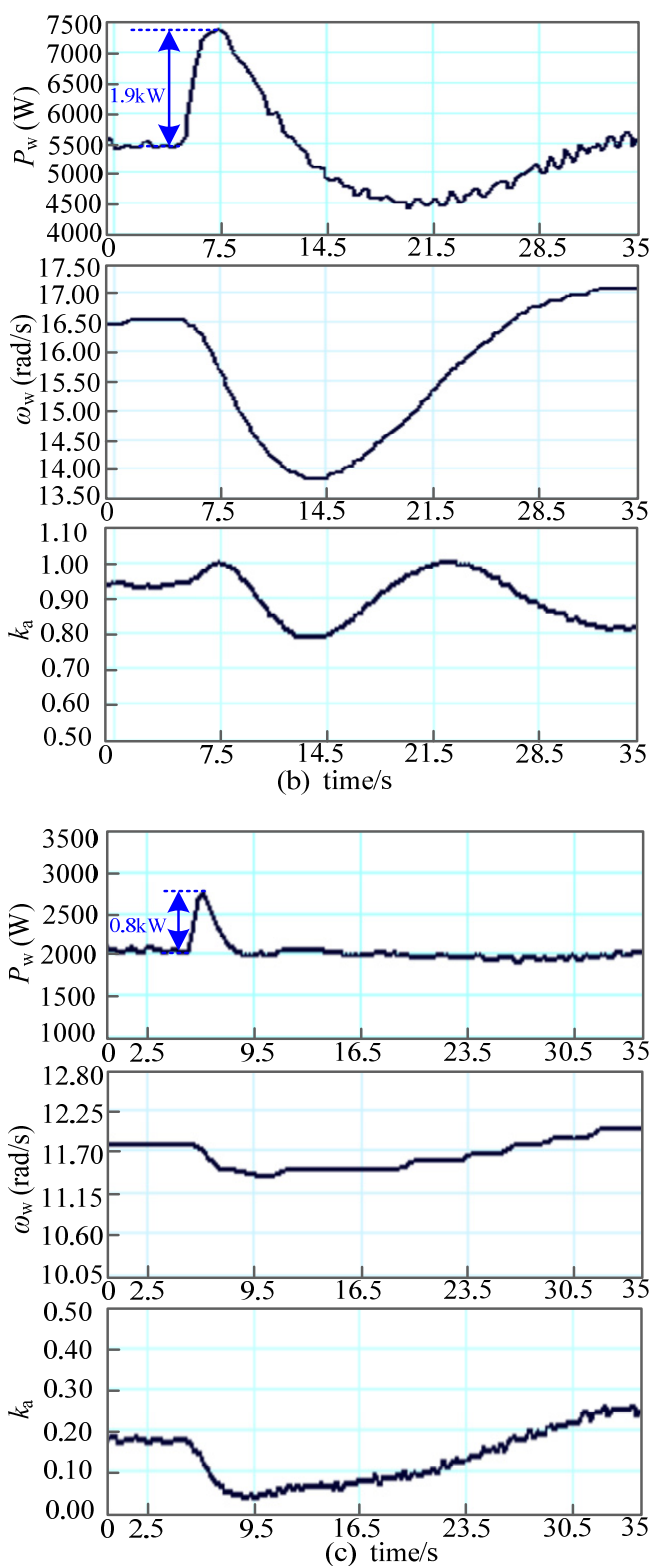

Fig. 13. System response characteristic curves with C-VIC introduced: (a) Characteristic curve of system frequency response; (b) Curves of rotating speed and output power of Simulative D-PMSG 1; (c) Curves of rotating speed and output power of Simulative D-PMSG 2. 
value of each being $1.1 \mathrm{~kW}$. In this case, Unit 2 has a quick decrease in its rotating speed and quits the system frequency response because of speed protection, whereas the Unit 1 fails to bring its frequency regulation capability into fully play.

\subsection{With C-VIC introduced}

The characteristic curve of system frequency response appear following the introduction of C-VIC, as shown in Fig. 13, similar to the simulation results in Fig. 7. The introduction of C-VIC into the system leads the lowest frequency to rise from $48.75 \mathrm{~Hz}$ to $49.27 \mathrm{~Hz}$. Considering the difference in frequency regulation capability between the units due to the introduction of frequency regulation coefficient into VIC, the auxiliary powers added to two simulative D-PMSGs are different. The maximum additional auxiliary power of Unit 1 increases to $1.9 \mathrm{~kW}$ whereas that of Unit 2 reduces to $0.8 \mathrm{~kW}$. As a result, the rotor kinetic energy of Unit 1 has been fully released while the amplitude of speed drop of Unit 2 have been reduced, thus to avoid triggering the speed protection. Besides, the speed recovery time of two units is different in process of frequency response, which can prevent the speed recovery from having a secondary effect on the system frequency.

\section{Conclusion}

Based on the researches into the method of calculating the parameters of the VIC and the method of assessing the frequency regulation capability of D-PMSGs, this paper has proposed a new C-VIC strategy by using the frequency regulation capability coefficient for D-PMSGs. This method is proved to be effective and feasible through simulations and experiments.

The analysis results show that the frequency regulation capability of D-PMSGs on the wind farm is related to their real-time operating states and is also limited by the rotor kinetic energy and capacity of their converters. The method of evaluating the frequency regulation capability of DPMSGs is used to deal with these two problems. Therefore, the proposed C-VIC strategy can bring the frequency regulation ability of D-PMSG into full play and avoid their excessive participation thus to effectively prevent a secondary drop in system frequency and frequent actions of the pitch angle control system.

Moreover, by considering the operating states of DPMSGs, the proposed C-VIC strategy enables the DPMSGs to have a certain automatic adjustment ability, which not only prevents the D-PMSGs from restoring their rotating speeds synchronously but also avoids communication between different D-PMSGs. Therefore, the design of the control system for a wind farm can be simplified.

\section{Acknowledgements}

This work was supported by the National Basic Research Program of China (973 Program) (No. 2012CB215103), and the National Natural Science Foundation of China (No. 51377167).

\section{Appendix}

\section{A. Parameters of the Simulation System}

Rated power of D-PMSG: $P_{w n}$ $2 \mathrm{MW}$

Rated rotating speed of wind turbine: $\omega_{w n} \quad 1.98 \mathrm{rad} / \mathrm{s}$

Blade radius of wind turbine: $r$

Rated wind speed of D-PMSG: $v_{n}$

Rotational inertia of D-PMSG: $J_{w}$

Inertia time constant of D-PMSG: $H_{w}$

$11.36 \mathrm{~m} / \mathrm{s}$

Inertia time constant of servo motor in pitch angle

control system: $T_{p}$

Lower limited value of pitch angle: $\theta_{\min }$ $6.63 \times 10^{6} \mathrm{~kg} \cdot \mathrm{m}^{2}$

Inertia time constant of the PMSG and the FPC: $T_{4} \quad 0.2 \mathrm{~s}$

Droop coefficient of D-PMSG: $R \quad 0.04$

Rated power of synchronous generator: $S_{n} \quad 60 \mathrm{MVA}$

Inertia time constant of synchronous generator: $H \quad 6 \mathrm{~s}$

Rated frequency of power grid: $f_{n}$

$50 \mathrm{~Hz}$

\section{B. Parameters of the experimental system}

Rated power of simulative D-PMSG: $P_{w n}$

$7.5 \mathrm{~kW}$

Rated rotating speed of wind turbine: $\omega_{w n}$

$18.8 \mathrm{rad} / \mathrm{s}$

Blade radius of wind turbine: $r \quad 3.4 \mathrm{~m}$

Rated wind speed: $v_{n} \quad 10 \mathrm{~m} / \mathrm{s}$

Inertia time constant of simulative D-PMSG: $H_{w} \quad 5.7 \mathrm{~s}$

Droop coefficient of simulative D-PMSG: $\quad R 0.04$

Rated power of synchronous generator: $S_{n} \quad 15 \mathrm{kVA}$

Inertia time constant of synchronous generator: $H \quad 3 \mathrm{~s}$

Rated frequency of power grid: $f_{n}$

$50 \mathrm{~Hz}$

\section{Nomenclature}

$P_{\text {wind }} \quad$ Wind power captured by the wind turbine (Watt).

$P_{w} \quad$ Grid-connected power of the D-PMSG (Watt).

$P_{M P P T} \quad$ Output power of maximum power point tracking (MPPT) control (Watt).

$P_{V I C} \quad$ Auxiliary output power of VIC (Watt).

$k_{\max } \quad$ Control coefficient of MPPT control.

$k_{p}, k_{d} \quad$ Control parameters of PDVIC.

$\Delta \omega^{*} \quad$ Per unit value of system frequency deviation.

$\omega \quad$ Angular frequency of the system $(\mathrm{rad} / \mathrm{s})$.

$\omega_{n} \quad$ Rated angular frequency of the power system $(\mathrm{rad} / \mathrm{s})$.

$J_{w} \quad$ Rotational inertia of the D-PMSG $\left(\mathrm{kg} \cdot \mathrm{m}^{2}\right)$. 


\begin{tabular}{|c|c|}
\hline$\omega_{w}$ & Angular velocity of the D-PMSG $(\mathrm{rad} / \mathrm{s})$. \\
\hline$\omega_{w 1}$ & $\begin{array}{l}\text { Minimum Angular velocity of D-PMSG } \\
\text { during VIC ( } \mathrm{rad} / \mathrm{s}) \text {. }\end{array}$ \\
\hline$\rho$ & Air density $\left(\mathrm{kg} / \mathrm{m}^{3}\right)$. \\
\hline$r$ & Blade radius $(\mathrm{m})$. \\
\hline$v$ & Wind speed $(\mathrm{m} / \mathrm{s})$. \\
\hline$C_{p}$ & Power coefficient. \\
\hline$\theta$ & Blade pitch angle (degree). \\
\hline$\lambda$ & Tip speed ratio. \\
\hline$R$ & Frequency droop coefficient. \\
\hline$p_{0 e}$ & $\begin{array}{l}\text { Number of pole pairs of the equivalent } \\
\text { synchronous generator. }\end{array}$ \\
\hline$H_{w}$ & Inertia time constant of D-PMSG (s). \\
\hline$J_{v i r}$ & $\begin{array}{l}\text { Rotational inertia of the equivalent } \\
\text { synchronous generator }\left(\mathrm{kg} \cdot \mathrm{m}^{2}\right) .\end{array}$ \\
\hline$H_{v i r}$ & $\begin{array}{l}\text { Inertia time constant of the equivalent } \\
\text { synchronous generator (s). }\end{array}$ \\
\hline$\gamma$ & Virtual inertia coefficient. \\
\hline$E_{k}$ & Kinetic energy of a wind power generator. \\
\hline$k_{J 1}, k_{J 2}$ & Available rotor kinetic energy factor. \\
\hline$k_{C 1}, k_{C 2}$ & Available capacity factor. \\
\hline$k_{a}, k_{a 1}, k_{a 2}$ & Frequency regulation capability coefficient. \\
\hline Subscripts & and Superscripts \\
\hline ref & Reference value. \\
\hline 0 & Initial value. \\
\hline$n$ & Rated value. \\
\hline$*$ & Per unit value. \\
\hline$\Delta$ & Deviation value. \\
\hline
\end{tabular}

\section{References}

[1] Monica Chinchilla, Santiago Arnaltes, Member, and Juan Carlos Burgos, "Control of permanent-magnet generators applied to variable-speed wind-energy systems connected to the grid," IEEE Trans. Power Syst., vol. 21, no. 1, pp. 130-135, Mar. 2006.

[2] Johan Morren, Jan Pierik, and Sjoerd W.H. de Haan, "Inertial response of variable speed wind turbines," Electric Power Systems Research, vol. 76, pp. 980987, 2006.

[3] Juan Manuel Mauricio, Alejandro Marano, Antonio Gómez-Expósito, and José Luis Martínez Ramos, "Frequency Regulation Contribution Through VariableSpeed Wind Energy Conversion Systems," IEEE Trans. Power Syst., vol. 24, no. 1, pp. 173-180, Feb. 2009.

[4] Yingcheng Xue, and Tai Nengling, "Review of contribution to frequency control through variable speed wind turbine," Renew. Energy, vol. 36, pp. 1671-1677, 2011.

[5] Xue Yingcheng, Tai Nengling, "Participation of doubly fed induction wind generators in system frequency regulation," IEEE Trans. Power Syst., vol. 22, no. 3, pp. 944-950, Aug. 2007.

[6] Barry G. Rawn, Peter W. Lehn, and Manfredi Maggiore, "Control Methodology to Mitigate the Grid Impact of Wind Turbines," IEEE Trans. Energy Convers., vol. 22, no. 2, pp.431-438, Jun. 2007.

[7] Rogério G. de Almeida, Edgardo D. Castronuovo, and J. A. Peças Lopes, "Optimum Generation Control in Wind Parks When Carrying Out System Operator Requests," IEEE Trans. Power Syst., vol. 22, no. 2, pp.718-725, May. 2006.

[8] Johan Morren, Sjoerd W. H. de Haan, Wil L. Kling, and J. A. Ferreira, "Wind Turbines Emulating Inertia and Supporting Primary Frequency Control," IEEE Trans. Power Syst., vol. 21, no. 1, pp. 433-434, Feb. 2006.

[9] Janaka Ekanayake and Nick Jenkins, "Comparison of the Response of Doubly Fed and Fixed-Speed Induction Generator Wind Turbines to Changes in Network Frequency," IEEE Trans. Energy Convers., vol. 19, no. 4, pp. 800-802, Dec. 2004.

[10] Le-Ren Chang-Chien, and Yao-Ching Yin, "Strategies for Operating Wind Power in a Similar Manner of Conventional Power Plant," IEEE Trans. Energy Convers., vol. 24, no. 4, pp. 926-934, Dec. 2009.

[11] K. V. Vidyanandan, and Nilanjan Senroy, "Primary Frequency Regulation by Deloaded Wind Turbines Using Variable Droop," IEEE Trans. Power Syst., vol. 28, no. 2, pp. 837-845, May. 2013.

[12] Ping-Kwan Keung, Pei Li, Hadi Banakar, and Boon Teck Ooi, "Kinetic Energy of Wind-Turbine Generators for System Frequency Support," IEEE Trans. Power Syst., vol. 24, no. 1, pp. 279-287, Feb. 2009.

[13] James F. Conroy, and Rick Watson, "Frequency Response Capability of Full Converter Wind Turbine Generators in Comparison to Conventional Generation," IEEE Trans. Power Syst., vol. 23, no. 2, pp. 649-656, May. 2008.

[14] Qiaoming Shi, Gang Wang, Lijun Fu, Lei Yuan, and He Huang, "State-space averaging model of wind turbine with PMSG and its virtual inertia control," in the 39th Annual Conference of the IEEE Industrial Electronics Society, Nov. pp. 1880-1886, 2013.

[15] Nayeem Rahmat Ullah, Torbjörn Thiringer, and Daniel Karlsson, "Temporary Primary Frequency Control Support by Variable Speed Wind Turbines Potential and Applications," IEEE Trans. Power Syst., vol. 23, no. 2, pp. 601-612, May. 2008.

[16] Hua Geng, Dewei Xu, Bin Wu, and Geng Yang, "Active Damping for PMSG-Based WECS with DCLink Current Estimation," IEEE Trans. Industrial Electronics, vol. 58, no. 4, pp. 1110-1119, Apr. 2011.

[17] Kundur Prabha, Power System Stability and Control, New York: McGraw-Hill, 1994.

[18] M. El Mokadem, V. Courtecuisse, C. Saudemont, B. 
Robyns, and J. Deuse, "Experimental study of variable speed wind generator contribution to primary frequency control," Renew. Energy, vol. 34, pp. 833844, 2009.

[19] Mostafa El Mokadem, Vincent Courtecuisse, Christophe Saudemont, Benoit Robyns, and Jacques Deuse, "Fuzzy Logic Supervisor-Based Primary Frequency Control Experiments of a Variable-Speed Wind Generator," IEEE Trans. Power Syst., vol. 24, no. 1, pp. 407-417, May. 2009.

[20] Qiaoming Shi, Gang Wang, Lijun Fu, Yang Liu, You $\mathrm{Wu}$, and $\mathrm{Li} \mathrm{Xu}$, "Virtual inertia control of D-PMSG based on the principle of active disturbance rejection control," Journal of Electrical Engineering \& Technology, vol. 10, no. 5, pp. 1969-1982, Jul. 2015.

[21] A. Mullane, G. Lightbody, R. Yacamini, "Windturbine fault ride through enhancement," IEEE Trans. Power Syst., vol. 20, no. 4, pp. 1929-1937, Nov. 2005.

[22] J. F. Conroy, and R. Watson, "Low-voltage ridethrough of a full converter wind turbine with permanent magnet generator," IET Renewable Power generation, vol. 1, no. 3, pp. 182-189, Sep. 2007.

[23] Qing-Chang Zhong, and George Weiss, "Synchronverters: Inverters That Mimic Synchronous Generators," IEEE Trans. Industrial Electronics, vol. 58, no. 4, pp. 1259-1267, Apr. 2011.

[24] Liansong Xiong, Fang Zhuo, Feng Wang, Xiaokang Liu, Ying Chen, and Minghua Zhu, "Static Synchronous Generator Model: A New Perspective to Investigate Dynamic Characteristics and Stability Issues of Grid-tied PWM Inverter," IEEE Trans. Power Electron., vol. 31, no. 9, pp. 6264-6280, Sept. 2016.

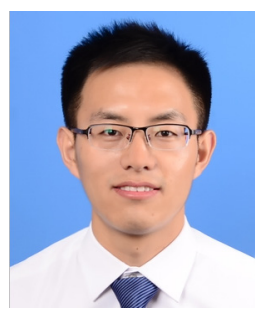

Qiaoming Shi received his B.S. and Ph.D. degrees from Xi'an Jiaotong University, China in 2010 and 2015 respectively. His research interests include the development of wind energy grid integration techniques.

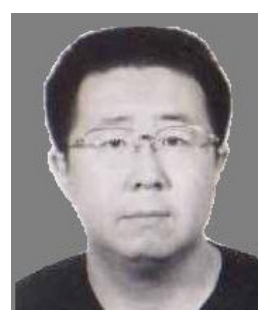

Gang Wang received his B.S. and Ph.D. degrees from Tsinghua University, China in 1993 and 2008 respectively. $\mathrm{He}$ is currently employed as Professor at Naval University of Engineering, China. His research field includes the development of stability control methods for wind power integrated system.

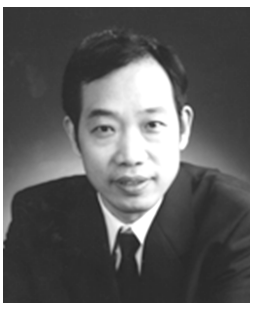

Weiming Ma received the M.S. degree in electrical engineering from the Naval University of Engineering in 1982 and the Ph.D. degree in electrical engineering from Tsinghua University in 1996. He was elected to the Chinese Academy of Engineering and became an academician in 2001. Now he is a professor in the Naval University of Engineering and also works as Director of National Key Laboratory for Vessel Integrated Power System.

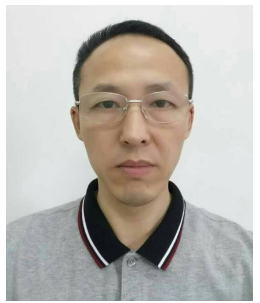

Lijun Fu received the B.S. degree in electrical engineering from Hunan University of Science and Technology in 1989, and the M.S. and Ph.D. degrees from Wuhan University, China respectively in 1995 and $1997 . \mathrm{He}$ is currently employed as Professor at Naval University of Engineering, China. His research field includes the development of stability control methods for Micro-grids.

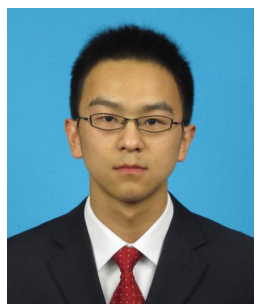

You Wu received his B.S. degree from Tsinghua University, China in 2013, and the M.S. degree from Naval University of Engineering, China in 2016. $\mathrm{He}$ is currently pursuing his Ph.D. degree at Naval University of Engineering. His research interests include the development of frequency control methods for wind power integrated system.

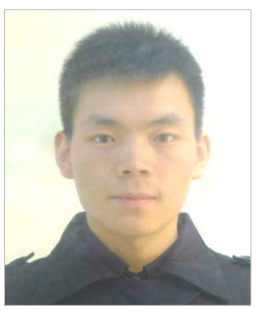

Pengxiang Xing received his B.S. degree in electrical engineering from Wuhan University, China in 2012. He is currently pursuing his Ph.D. degree in the School of Electrical Engineering, Wuhan University. His research interests include renewable energy generation and micro-grid. 